\title{
In Silico Analysis Identifies Differently Expressed lncRNAs as Novel Biomarkers for the Prognosis of Thyroid Cancer
}

\author{
Yuansheng Rao, Haiying Liu, Xiaojuan Yan, and Jianhong Wang $(\mathbb{D}$ \\ Department of Otorhinolaryngology, Beijing Anzhen Hospital, No. 2 Anzhen Road, Chaoyang District, Beijing 100029, China \\ Correspondence should be addressed to Jianhong Wang; ou70394137@163.com
}

Received 3 February 2020; Accepted 24 March 2020; Published 23 April 2020

Guest Editor: Tao Huang

Copyright @ 2020 Yuansheng Rao et al. This is an open access article distributed under the Creative Commons Attribution License, which permits unrestricted use, distribution, and reproduction in any medium, provided the original work is properly cited.

\begin{abstract}
Background. Thyroid cancer (TC) is one of the most common type of endocrine tumors. Long noncoding RNAs had been demonstrated to play key roles in TC. Material and Methods. The lncRNA expression data were downloaded from Co-lncRNA database. The raw data was normalized using the limma package in $\mathrm{R}$ software version 3.3.0. The differentially expressed $\mathrm{mRNA}$ and $\operatorname{lncRNAs}$ were identified by the linear models for the microarray analysis (Limma) method. The DEGs were obtained with thresholds of $|\log \mathrm{FC}|>1.5$ and $P<0.001$. The hierarchical cluster analysis of differentially expressed mRNAs and lncRNAs was performed using CLUSTER 3.0, and the hierarchical clustering heat map was visualized by Tree View. Results. In the present study, we identified 6 upregulated and 85 downregulated lncRNAs in TC samples. Moreover, we for the first time identified 16 downregulated lncRNAs was correlated to longer disease-free survival time in patients with TC, including ATP1A1-AS1, CATIP-AS1, FAM13A-AS1, LINC00641, LINC00924, MIR22HG, NDUFA6-AS1, RP11-175K6.1, RP11-727A23.5, RP11774O3.3, RP13-895J2.2, SDCBP2-AS1, SLC26A4-AS1, SNHG15, SRP14-AS1, and ZNF674-AS1. Conclusions. Bioinformatics analysis revealed these lncRNAs were involved in regulating the RNA metabolic process, cell migration, organelle assembly, tRNA modification, and hormone levels. This study will provide useful information to explore the potential candidate biomarkers for diagnosis, prognosis, and drug targets for TC.
\end{abstract}

\section{Introduction}

Thyroid cancer (TC) is one of the most common type of endocrine tumors [1]. A recent study showed the incidence of TC increased rapidly worldwide, especially in female. However, there was still lacking of effective biomarkers for the prognosis of TC. Over the past decades, several genes were identified to be related to the progression of TC and could serve as potential biomarkers for TC, such as RAS [2] and BRAF (V600E) [3] gene mutations. Moreover, with the development of the next-generation sequencing method, a series of public datasets were developed to explore the potential biomarkers and mechanisms underlying tumor progression in human cancers. For example, Wang et al. analyzed TCGA dataset and found IncRNA UNC5B-AS1 promoted TC growth and metastasis [4]. Identification of novel biomarkers is still an urgent need for the TC.

Long noncoding RNAs (lncRNAs) were reported to play important roles in tumorigenesis and cancer progression [5].
LncRNAs bound to chromatin, proteins, and RNAs to modulate cancer proliferation, apoptosis, autophagy, epithelialmesenchymal transition (EMT), and metastasis [6]. In TC, ENST00000539653 promoted cancer progression via MAPK signaling. TUG1 regulated TC cell proliferation and EMT through targeting miR-145 [7]. A recent study showed antisense lncRNA COMET repression inhibited cell viability and invasiveness and induced sensitivity to vemurafenib in BRAF- and RET-driven TC [8]. Interestingly, emerging studies demonstrated lncRNAs could serve as potential prognostic or diagnostic biomarkers for human cancers. For instance, Zhang et al. reported that downregulation of DANCR is a biomarker for TC diagnosis [9]. Decreased EMX2OS expression was associated with unfavorable recurrence-free survival (RFS) in classical PTC [10].

In this study, we identified differently expressed lncRNAs using two public datasets, including Co-lncRNA database and GEPIA database [11]. Then, coexpression network analysis, gene ontology (GO) analysis, and Kyoto Encyclopedia of 


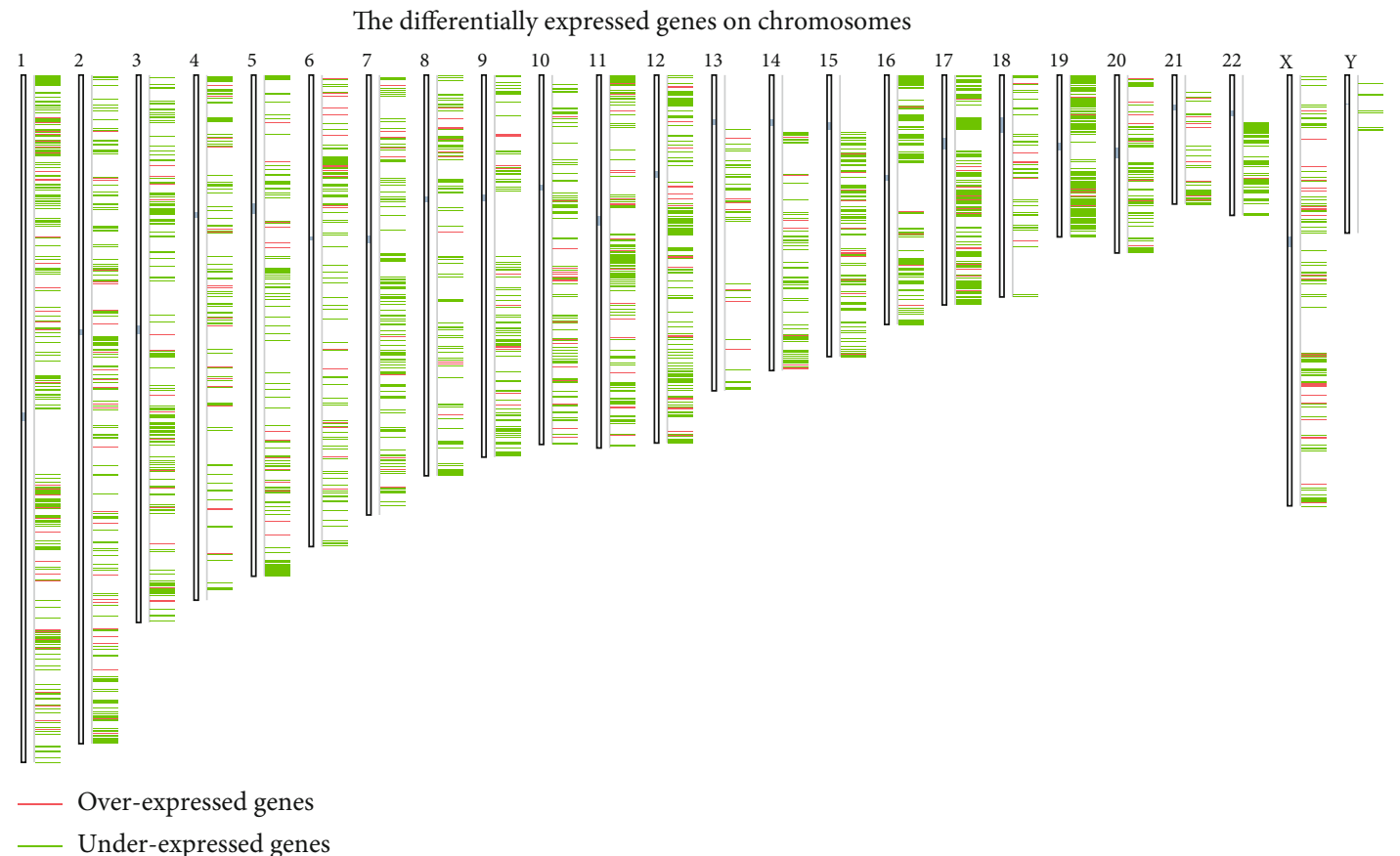

(a)

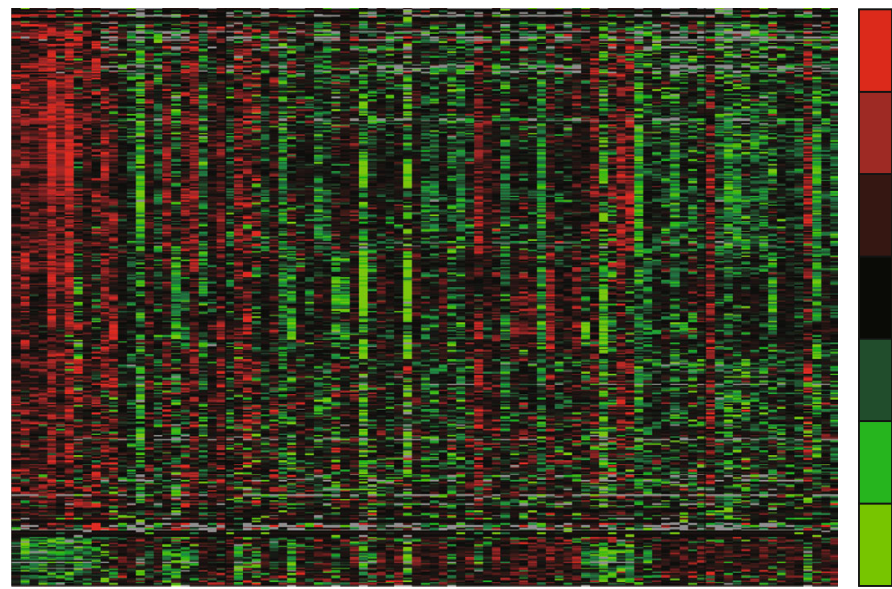

(b)
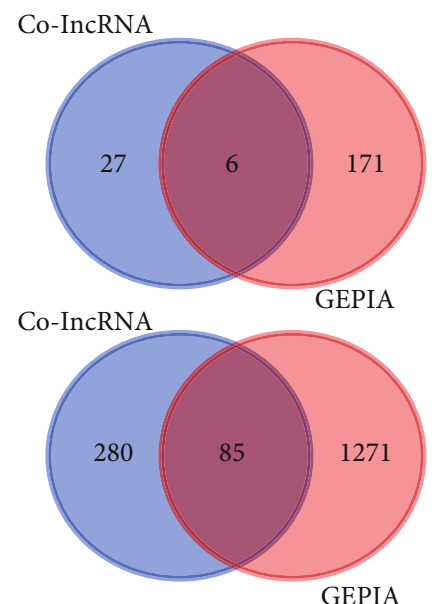

(c)

Figure 1: Identification of differently expressed lncRNAs in TC. (a) Chromosomal distribution of differently expressed genes in TC tissues using GEPIA database. (b) Hierarchical clustering analysis shows differential lncRNA expression between normal and TC samples by using Co-lncRNA database. (c, d) Venn diagrams display differently expressed lncRNAs in both databases.

Genes and Genomes (KEGG) pathway analysis were used to evaluate the potential functions of these lncRNAs in TC. We thought this study could provide novel biomarkers for TC.

\section{Materials and Methods}

2.1. Public Dataset Analysis. The lncRNA expression data were downloaded from Co-lncRNA database. Co-lncRNA database included 12 normal samples and 83 TC samples. The raw data was normalized using the limma package in $\mathrm{R}$ software version 3.3.0 (https://www.r-project.org/). The differentially expressed mRNA and lncRNAs were identified by the linear models for microarray analysis (Limma) method [12]. The DEGs were obtained with thresholds of $|\log \mathrm{FC}|>1.5$ and $P<0.001$. The hierarchical cluster analysis of differentially expressed mRNAs and lncRNAs was performed using CLUSTER 3.0 [13], and the hierarchical clustering heat map was visualized by Tree View [14].

2.2. Coexpression Network Construction and Analysis. In this study, as $\mathrm{Hu}$ et al. [15] described, the Pearson correlation coefficient of DEG-lncRNA pairs was calculated according to the expression value of them. The coexpressed DEGlncRNA pairs with the absolute value of Pearson correlation coefficient $\geq 0.75$ were selected, and the coexpression network was established by using cytoscape software. Cytoscape 


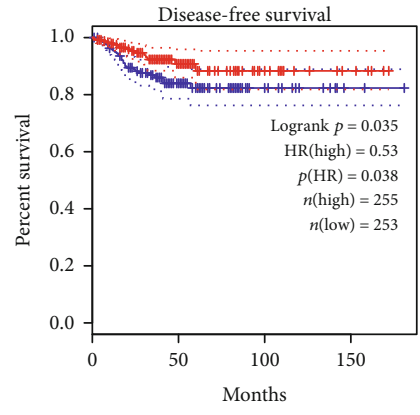

— Low ATP1A1-AS1 TPM — High ATP1A1-AS1 TPM

(a)

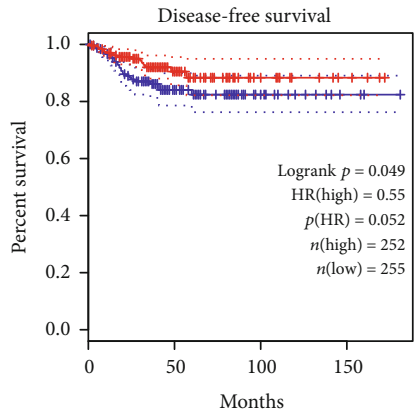

_ Low LINC00641-AS1 TPM

_ High LINC00641-AS1 TPM

(d)

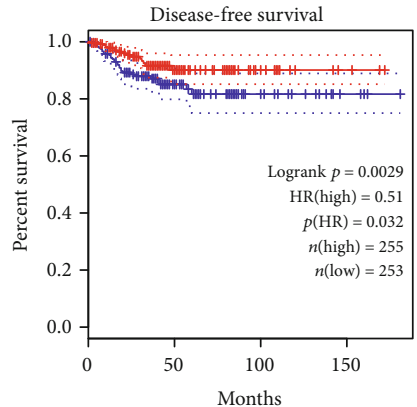

_ Low NDUFA6-AS1 TPM

— High NDUFA6-AS1 TPM

(g)

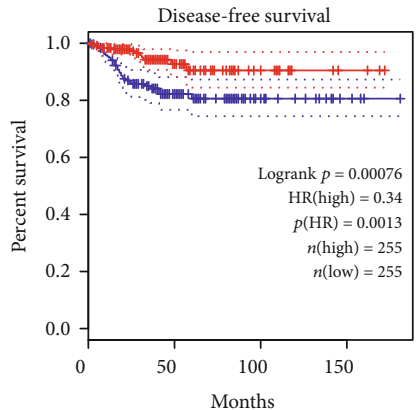

Low RP11-77403.3 TPM

— High RP11-774O3.3 TPM

(j)

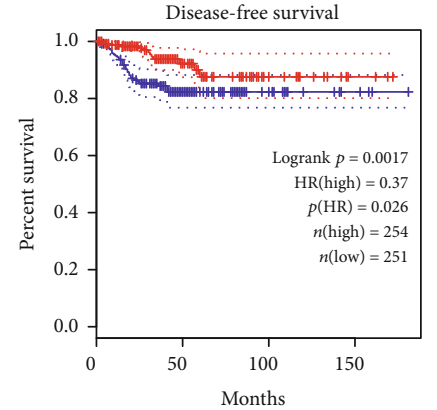

L Low ATP1A1-AS1 TPM

— High ATP1A1-AS1 TPM

(b)

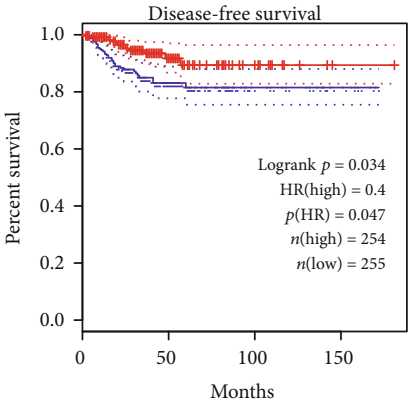

—— Low LINC00924 TPM TPM

— High LINC00924 TPM TPM

(e)

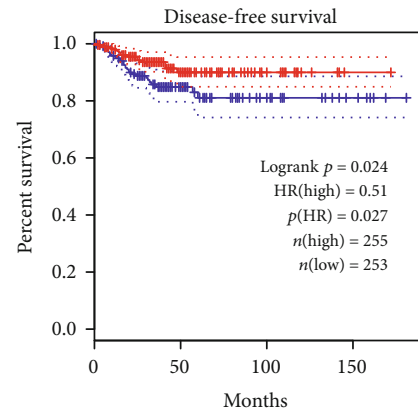

__ Low RP11-175K6.1 TPM

— High RP11-175K6.1 TPM

(h)

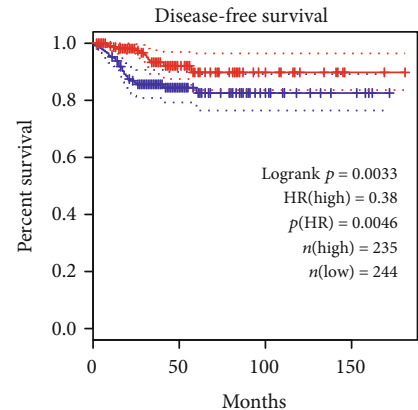

\begin{tabular}{l} 
Low RP11-895J2.2 TPM \\
\hline High RP11-895J2.2 TPM
\end{tabular}

(k)

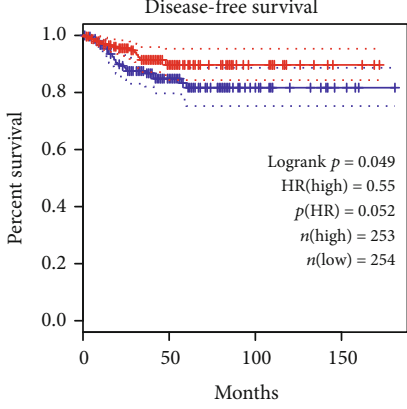

— Low FAM13A-AS1 TPM

— High FAM13A-AS1 TPM

(c)

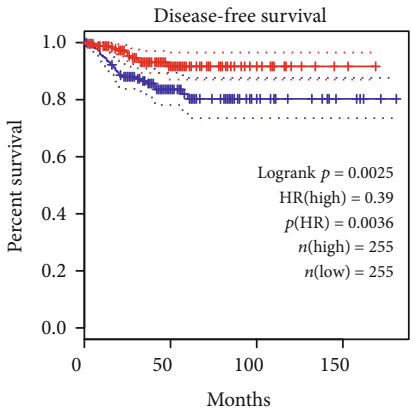

— Low MIR22HG TPM

High MIR22HG TPM

(f)

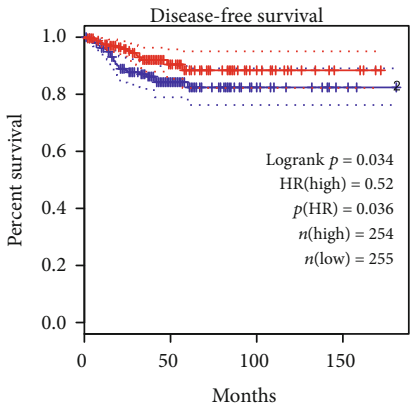

—_ Low RP11-727A23.5 TPM

— High RP11-727A23.5 TPM

(i)

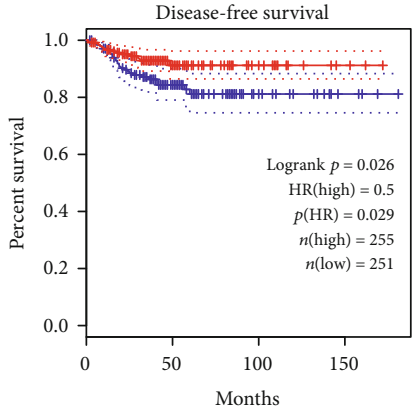

Low SDCBP2-AS1 TPM

— High SDCBP2-AS1 TPM

(1)

FIgure 2: Continued. 


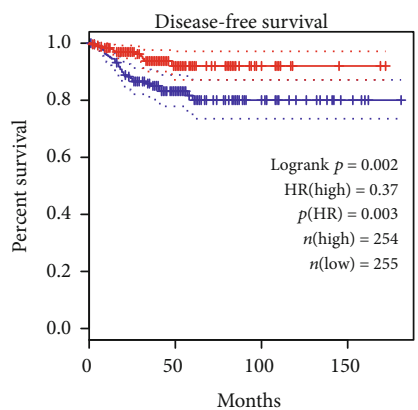

_ Low SLC26A4-AS1 TPM

$(\mathrm{m})$

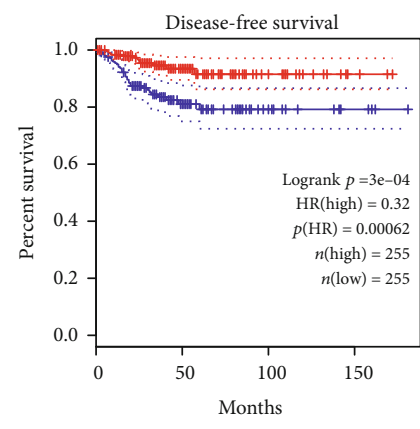

L Low SNHG15 TPM

— High SNHG15 TPM

(n)
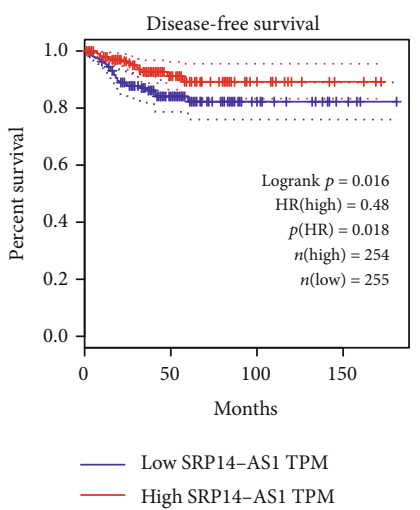

(o)

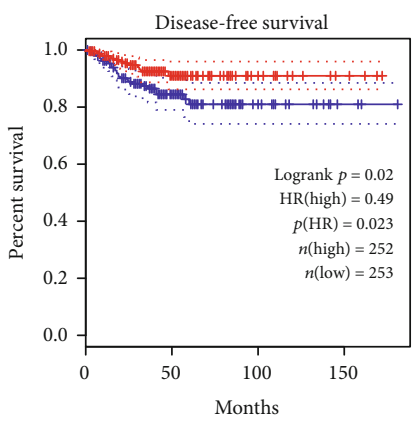

Low ZNF674-AS1 TPM — High ZNF674-AS1 TPM

(p)

FIGURE 2: Identification of disease-free survival time related lncRNAs in TC. (a-p) Higher expression levels of ATP1A1-AS1 (a), CATIP-AS1 (b), FAM13A-AS1 (c), LINC00641 (d), LINC00924 (e), MIR22HG (f), NDUFA6-AS1 (g), RP11-175K6.1 (h), RP11-727A23.5 (i), RP11774 O3.3 (j), RP13-895J2.2 (k), SDCBP2-AS1 (l), SLC26A4-AS1 (m), SNHG15 (n), SRP14-AS1 (o), and ZNF674-AS1 (p) were significantly correlated to longer disease-free survival time in patients with TC.

MCODE plug-in (version 3.4.0, available online: http://www .cytoscape.org/) was applied for visualization of the coexpression networks.

Gene coexpression analysis could be applied to related genes of unknown function with GO or to analysis candidate disease genes or to predict transcriptional regulatory mechanism [16].

2.3. GO and KEGG Pathway Analyses. To identify functions of DEGs in smoking-related lung cancer, we performed GO function enrichment analysis in 3 functional ontologies: biological process (BP), cellular component (CC), and molecular function (MF). KEGG pathway enrichment analysis was also performed to identify pathways enriched in smoking-related lung cancer using the DAVID system (https://david.ncifcrf.gov/). The $P$ value less than 0.05 was considered significant.

2.4. Survival Analysis. GEPIA database (http://gepia.cancerpku.cn/index.html) was used to predict the correlation between candidate gene expression and overall survival (OS) time or disease-free survival (DFS) time. The median expression of target was selected as cutoff to divide all TC samples as high and low groups. The probability of survival was estimated using the Kaplan-Meier method. The logrank test was used to compare differences in survival times.

2.5. Statistical Analysis. The numerical data were presented as mean \pm standard deviation (SD) of at least three determinations. Statistical comparisons between groups of normalized data were performed using $T$-test or Mann-Whitney $U$ test according to the test condition. A $P<0.05$ was considered statistical significance with a $95 \%$ confidence level.

\section{Results}

3.1. Identification of Differently Expressed IncRNAs in TC. GEPIA database was first analyzed. Our results identified 177 upregulated lncRNAs and 1359 downregulated lncRNAs in TC samples compared to normal tissues (Figure 1(a) and
Supplementary Table 1). By analyzing Co-lncRNA database, 399 lncRNAs were found to be dysregulated in TC. Among these lncRNAs, 33 lncRNAs were overexpressed and 366 lncRNAs were suppressed in TC tissues compared to normal tissues (Figure 1(b)).

By performing integrated analysis of Co-lncRNA and GEPIA databases, a total of $6 \operatorname{lncRNAs}$ were found to be upregulated and 85 lncRNAs were found to be downregulated in TC samples (Figure 1(c)). CATIP-AS1 is the most significantly downregulated lncRNA, and RP11-280O1.2 is the most significantly upregulated lncRNA in TC.

3.2. Downregulated IncRNAs Were Correlated to Longer Disease-Free Survival Time in TC. Then, the GEPIA dataset was used to explore the correlation between lncRNA expression and overall survival time in TC. The median expression level of target gene was selected as the cutoff to divide all TC samples into high and low groups. Our analyses showed dysregulated lncRNAs were significantly correlated to the disease-free survival time in TC. Higher expression levels of ATP1A1-AS1, CATIP-AS1, FAM13A-AS1, LINC00641, LINC00924, MIR22HG, NDUFA6-AS1, RP11175K6.1, RP11-727A23.5, RP11-774O3.3, RP13-895J2.2, SDCBP2-AS1, SLC26A4-AS1, SNHG15, SRP14-AS1, and ZNF674-AS1 were significantly correlated to longer diseasefree survival time in patients with TC (Figures 2(a)-2(p)).

Of note, we found that ATP1A1-AS1, CATIP-AS1, FAM13A-AS1, LINC00641, LINC00924, MIR22HG, NDUFA6-AS1, RP11-175K6.1, RP11-727A23.5, RP11774O3.3, RP13-895J2.2, SDCBP2-AS1, SLC26A4-AS1, SNHG15, SRP14-AS1, and ZNF674-AS1 were significantly downregulated in TC samples compared to normal tissues (Figures 3(a)-3(p)). These results suggested these lncRNAs may serve as tumor suppressors in TC.

3.3. Construction of Differently Expressed lncRNAs Regulating Coexpression Network in TC. Furthermore, we constructed differently expressed lncRNAs regulating coexpression network in TC. The Pearson correlation coefficients between 


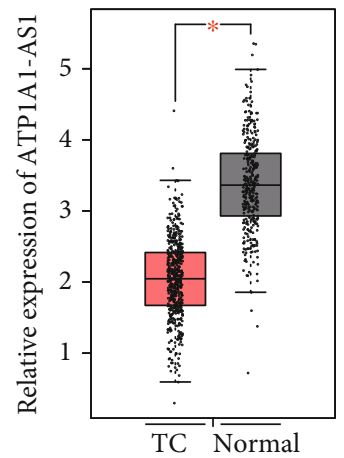

(a)

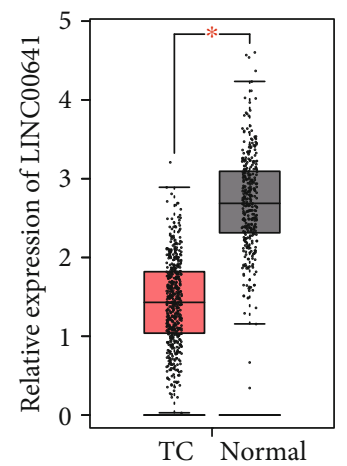

(d)

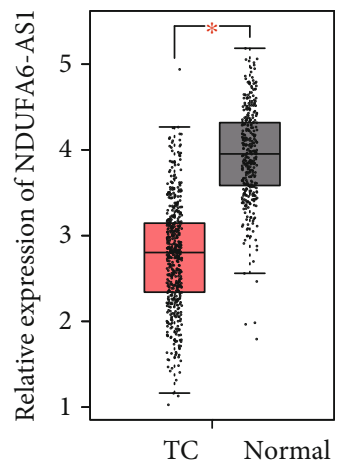

(g)

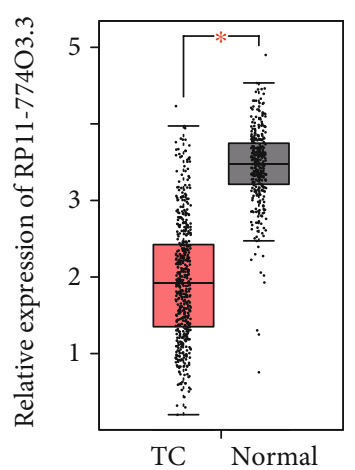

(j)

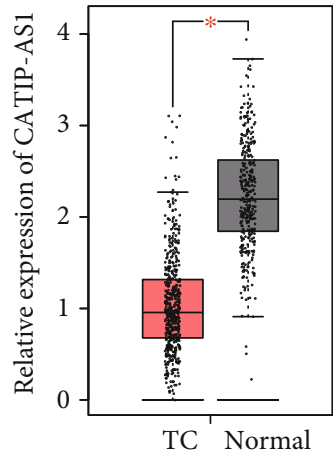

(b)

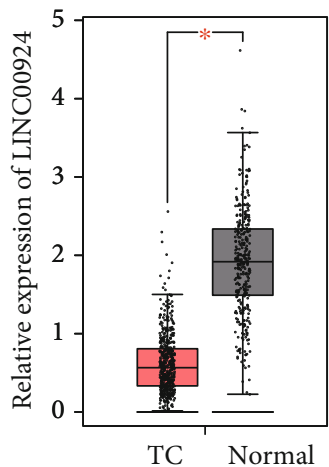

(e)

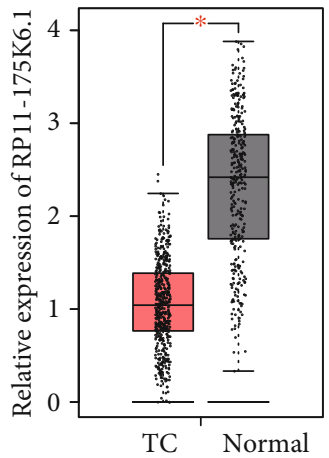

(h)

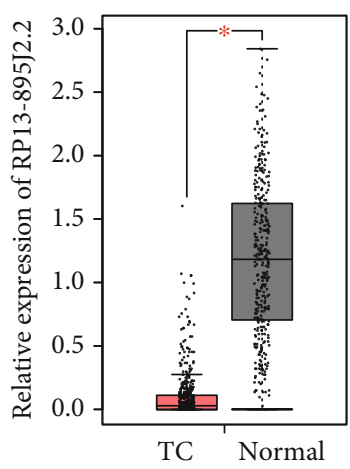

(k)

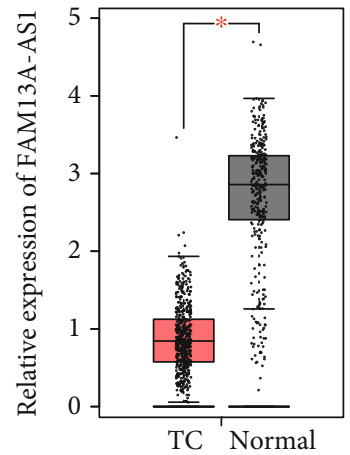

(c)

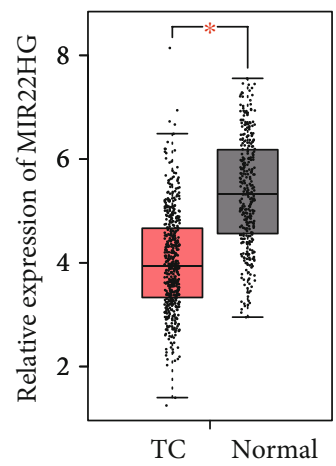

(f)

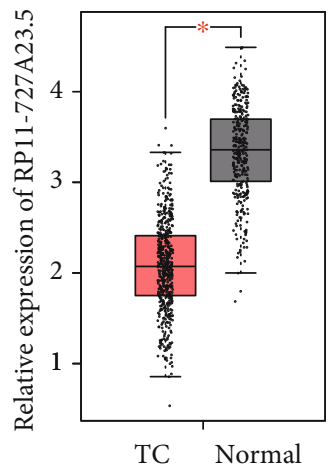

(i)

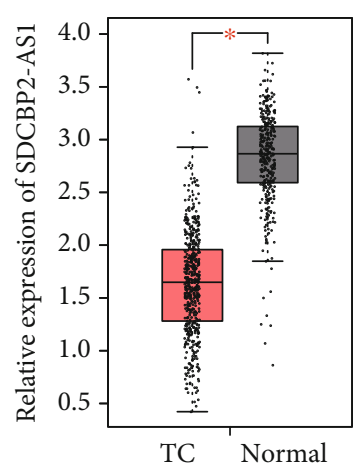

(l)

Figure 3: Continued. 


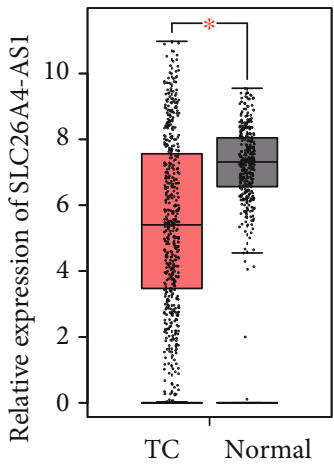

$(\mathrm{m})$

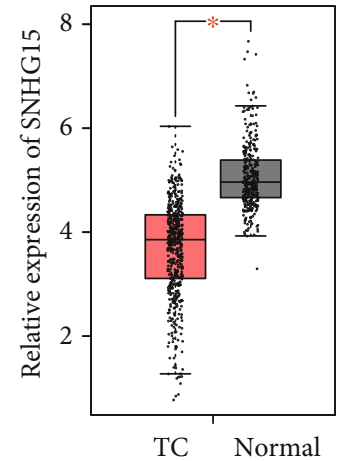

(n)

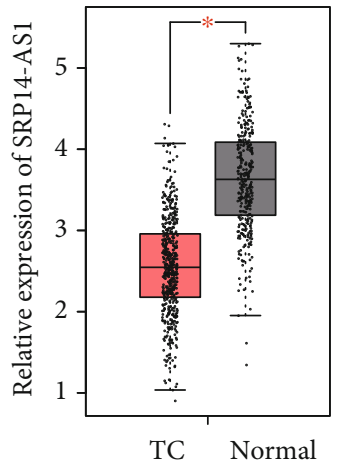

(o)

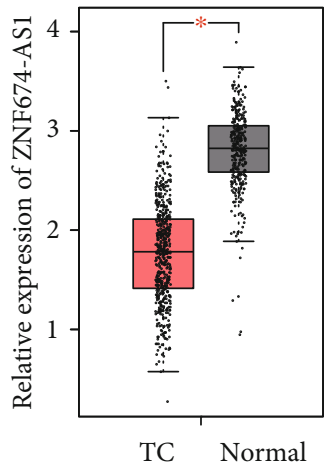

(p)

Figure 3: We identified downregulated lncRNAs in TC. (a-p) The expression levels of ATP1A1-AS1 (a), CATIP-AS1 (b), FAM13A-AS1 (c), LINC00641 (d), LINC00924 (e), MIR22HG (f), NDUFA6-AS1 (g), RP11-175K6.1 (h), RP11-727A23.5 (i), RP11-774O3.3 (j), RP13-895J2.2 (k), SDCBP2-AS1 (l), SLC26A4-AS1 (m), SNHG15 (n), SRP14-AS1 (o), and ZNF674-AS1 (p) were downregulated in TC samples compared to normal tissues.

lncRNA and mRNA was downloaded from GEPIA datasets. We selected the top 200 correlated genes as the potential targets of differently expressed lncRNAs. As shown in Figure 4, we found that this coexpression network contained 16 lncRNAs and 2698 mRNAs.

3.4. Bioinformatics Analysis of Differently Expressed lncRNAs in TC. Furthermore, we performed bioinformatics analysis for differentially expressed lncRNAs in TC (Figure 5). GO analysis showed that ATP1A1-AS1 [17] was involved in regulating the RNA metabolic process, the nucleic acid metabolic process, regulation of gene expression, transcription, DNA-templated, and cellular macromolecule metabolic process. FAM13A-AS1 [18] was involved in regulating RNA splicing and mRNA processing. LINC00641 was involved in regulating the RNA metabolic process; gene expression; mRNA processing; regulation of gene expression; RNA splicing; and mRNA splicing, via spliceosome, and transcription [19]. LINC00924 was associated with the regulation of cell migration, regulation of cellular component movement, regulation of locomotion, cell adhesion, circulatory system development, and locomotion. MIR22HG was involved in regulating organelle assembly, positive regulation of the RNA metabolic process, cilium organization, axoneme assembly, and regulation of gene expression [20]. RP11$175 \mathrm{~K} 6.1$ was involved in regulating vasculature development, blood vessel development, circulatory system development, blood vessel morphogenesis, angiogenesis, and tube morphogenesis. RP11-727A23.5 was involved in regulating mRNA processing, RNA splicing, inner dynein arm assembly, cilium assembly, and gene expression. SDCBP2-AS1 was involved in regulating the tRNA process, tRNA methylation, methylation, macromolecule methylation, and tRNA modification [21]. SLC26A4-AS1 was involved in regulating regulation of hormone levels, the oxidation-reduction process, thyroid hormone generation, the hormone metabolic process, and the alpha-amino acid metabolic process [22]. SRP14-AS1 was involved in regulating cilium movement, determination of left/right symmetry, photoreceptor cell outer segment organization, inner dynein arm assembly, and organelle assembly (Figure 5(a)-5(j)).

\section{Discussion}

Thyroid cancer is a rare but a highly lethal form of thyroid cancer, which needs more attention. And lncRNAs had been demonstrated to play key roles in the progression of most human cancers, including thyroid cancer. For instance, DGCR5 played as a tumor suppressor in TC though binding to miR-2861 [23]. SNHG16 promoted TC proliferation and invasion through modulation of miR-497 [24]. GAS8-AS1 inhibited TC growth through miR-135b-5p/CCND2 axis. Of note, lncRNAs were also found to be dysregulated in TC, suggesting the potential prognostic value of lncRNAs. For example, a bioinformatics analysis study showed that FAM95B1 and UCA1 were correlated with cervical lymph node metastasis, tumor staging, and TC prognosis. Lu et al. reported that the dysregulation of RUNDC3A-AS1, FOXDAS1, RUNDC3A-AS1 and FOXD-AS1 was correlated to a shorter overall survival time in patients with TC [25]. However, only a small part of lncRNAs were reported in TC. The expression pattern and molecular functions of most lncRNAs in TC remained unknown.

In our study, silico analyses were performed to identify TC-related important lncRNA. Co-lncRNA and GEPIA databases were used to identify differently expressed lncRNAs in TC. There are a total of 6 upregulated and 85 downregulated lncRNAs in TC samples compared to normal tissues. Among these lncRNAs, only few lncRNAs were reported in previous studies. For example, NR2F1-AS1 was found to be upregulated in TC samples. In hepatocellular carcinoma, knockdown of NR2F1-AS1 significantly suppressed cancer invasion, migration, and in vivo tumor growth [26]. Moreover, we for the first time identified 16 downregulated IncRNAs was correlated to a longer disease-free survival time in patients with TC, including ATP1A1-AS1, CATIP-AS1, FAM13A-AS1, LINC00641, LINC00924, MIR22HG, NDUFA6-AS1, RP11-175K6.1, 


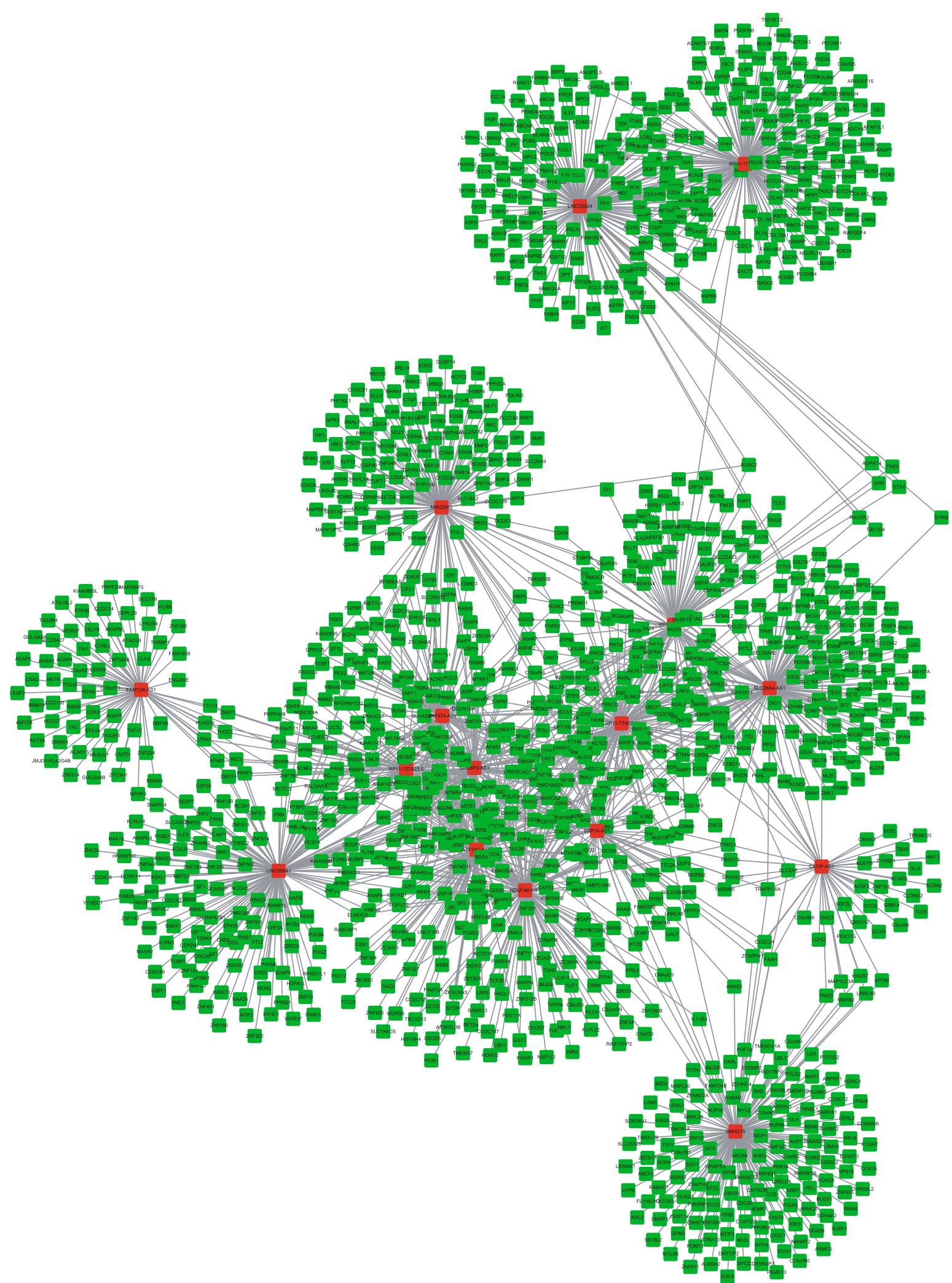

Figure 4: Coexpression network analysis of lncRNAs in TC. Coexpression network analysis of lncRNAs in TC. Red nodes: lncRNA; green nodes: mRNA. 


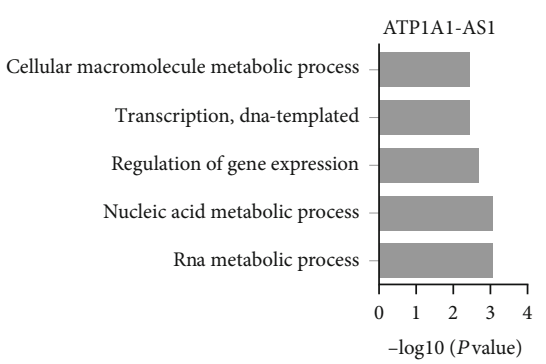

(a)

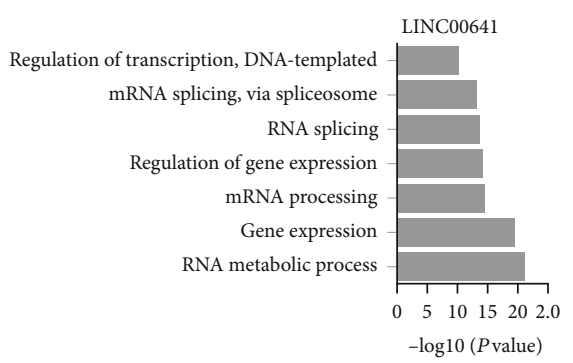

(c)

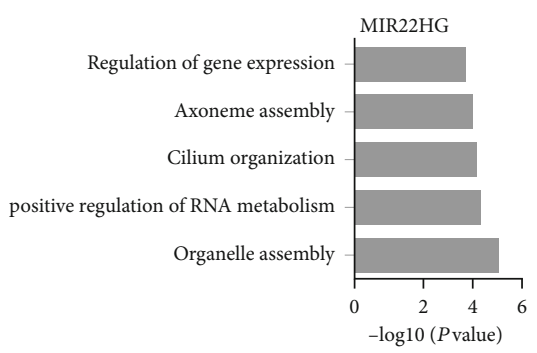

(e)

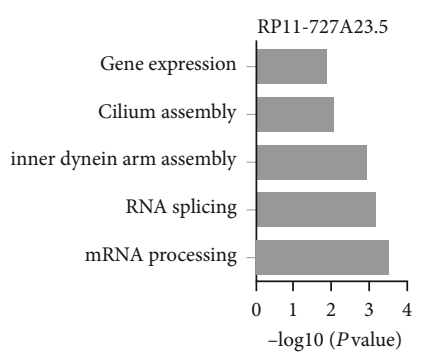

(g)

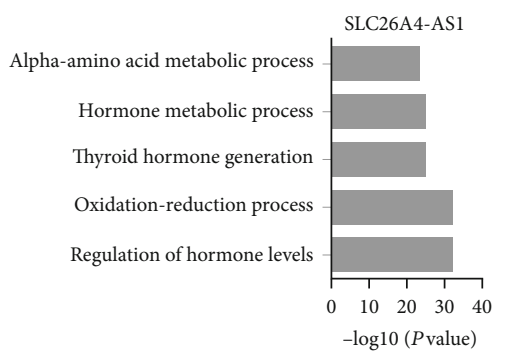

(i)

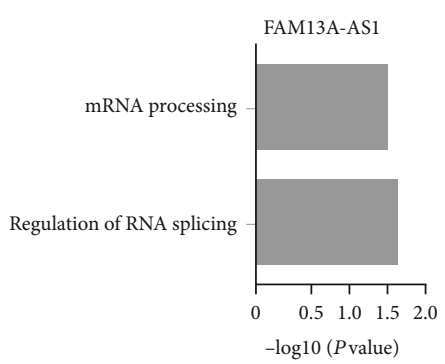

(b)

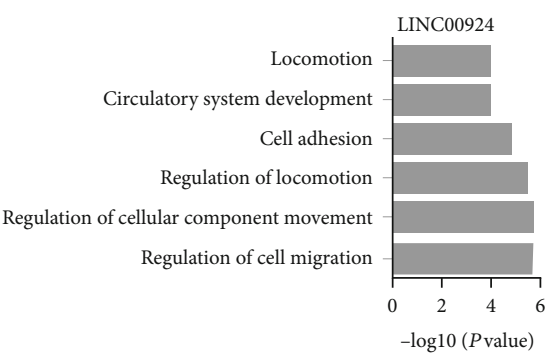

(d)

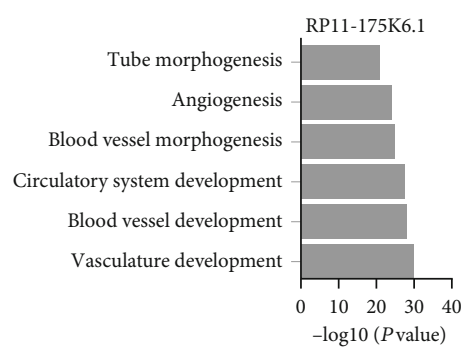

(f)

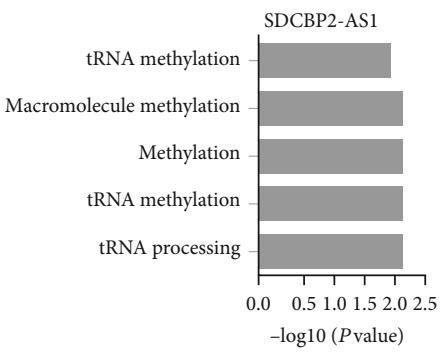

(h)

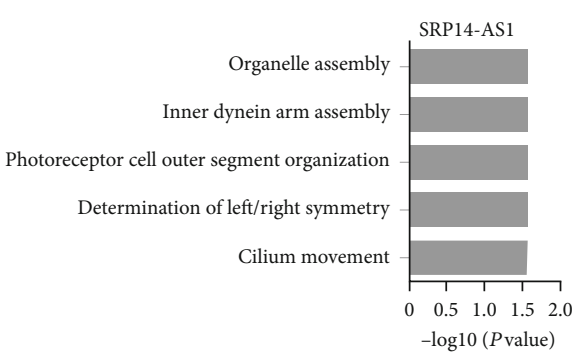

(j)

FIGURE 5: Bioinformatics analysis of differently expressed lncRNAs. (a-j) Bioinformatics analysis of ATP1A1-AS1 (a), FAM13A-AS1 (b), LINC00641 (c), LINC00924 (d), MIR22HG (e), RP11-175K6.1 (f), RP11-727A23.5 (g), SDCBP2-AS1 (h), SLC26A4-AS1 (i), and SRP14AS1 $(j)$ in TC.

RP11-727A23.5, RP11-774O3.3, RP13-895J2.2, SDCBP2AS1, SLC26A4-AS1, SNHG15, SRP14-AS1, and ZNF674AS1. The functions of these lncRNAs remained unclear.
ATP1A1-AS1 is a novel lncRNA. A previous study showed ATP1A1-AS1 is a negative regulator of $\mathrm{Na} / \mathrm{K}$-ATPase $\alpha 1$ and involved in regulating cell proliferation in human 
kidney cells. LINC00641 was reported as a tumor suppressor in bladder cancer via sponging miR-197 [19]. SLC26A4-AS1was found to be associated with overall survival in gastric cancer. SNHG15 was reported to be downregulated in thyroid cancer and acted as a tumor suppressor in TC [27].

LncRNA coexpression network was widely used to explore the potential roles of novel lncRNAs in TC. For example, Zhang et al. revealed that HCG11 was involved in regulating the MAPK signaling pathway and gene transcription though coexpression analysis [28]. In this study, we constructed a network including 19 downregulated lncRNAs and 2698 mRNAs. Bioinformatics analysis showed these lncRNAs played crucial roles in TC progression. For example, ATP1A1-AS1, RP11-727A23.5, and LINC00641 were involved in regulating the RNA metabolic process. FAM13A-AS1 was involved in regulating RNA splicing. LINC00924 was associated with the regulation of cell migration and cell adhesion. MIR22HG was involved in regulating organelle assembly. RP11-175K6.1 was involved in regulating vasculature development. SDCBP2-AS1 was involved in regulating tRNA modification. SLC26A4-AS1 was involved in regulating hormone levels.

In conclusion, we identified 6 upregulated and 85 downregulated lncRNAs in TC samples. Moreover, we for the first time identified 16 downregulated lncRNAs was correlated to a longer disease-free survival time in patients with TC, including ATP1A1-AS1, CATIP-AS1, FAM13AAS1, LINC00641, LINC00924, MIR22HG, NDUFA6-AS1, RP11-175K6.1, RP11-727A23.5, RP11-774O3.3, RP13895J2.2, SDCBP2-AS1, SLC26A4-AS1, SNHG15, SRP14AS1, and ZNF674-AS1. Bioinformatics analysis revealed these lncRNAs were involved in regulating the RNA metabolic process, cell migration, organelle assembly, tRNA modification, and hormone levels. This study will provide useful information to explore the potential candidate biomarkers for diagnosis, prognosis, and drug targets for TC.

\section{Abbreviations}

$\begin{array}{ll}\text { TC: } & \text { Thyroid cancer } \\ \text { Lnc-RNAs: } & \text { Long noncoding RNAs } \\ \text { EMT: } & \text { Epithelial-mesenchymal transition } \\ \text { RFS: } & \text { Recurrence-free survival } \\ \text { GO: } & \text { Gene Ontology } \\ \text { KEGG: } & \text { Kyoto Encyclopedia of Genes and Genomes } \\ \text { BP: } & \text { Biological process } \\ \text { CC: } & \text { Cellular component } \\ \text { MF: } & \text { Molecular function } \\ \text { OS: } & \text { Overall survival } \\ \text { DFS: } & \text { Disease-free survival. }\end{array}$

\section{Data Availability}

All the data in this manuscript can be accessed in CoLncRNA (http://www.biobigdata.com/Co-LncRNA/).

\section{Ethical Approval}

All procedures performed in studies involving human participants were in accordance with the ethical standards of the institutional and/or national research committee and with the 1964 Helsinki declaration and its later amendments or comparable ethical standards.

\section{Conflicts of Interest}

The authors declare that they have no conflict of interest.

\section{Authors' Contributions}

Jianhong Wang conceived and designed the study. Yuansheng Rao and Haiying Liu were responsible for the collection and analysis of the data. All authors interpreted the data, drafted the manuscript, and approved the final version of the manuscript.

\section{Supplementary Materials}

Supplementary table 1: the differently expressed lncRNAs in TC by using GEPIA database. (Supplementary Materials)

\section{References}

[1] A. S. Al-Zahrani and K. Ravichandran, "Epidemiology of thyroid cancer: a review with special reference to Gulf Cooperation Council (GCC) states," The Gulf Journal of Oncology, vol. 2, pp. 17-28, 2007.

[2] J. L. Bos, "Ras oncogenes in human cancer: a review," Cancer Research, vol. 49, no. 17, p. 4682, 1989.

[3] D. Dankort, E. Filenova, M. Collado, M. Serrano, K. Jones, and M. McMahon, "A new mouse model to explore the initiation, progression, and therapy of BRAFV600E-induced lung tumors," Genes \& Development, vol. 21, no. 4, pp. 379-384, 2007.

[4] Y. Wang, A. Bhandari, J. Niu et al., "The lncRNA UNC5B-AS1 promotes proliferation, migration, and invasion in papillary thyroid cancer cell lines," Human Cell, vol. 32, no. 3, pp. 334-342, 2019.

[5] M. Cong, J. Li, R. Jing, and Z. Li, "Long non-coding RNA tumor suppressor candidate 7 functions as a tumor suppressor and inhibits proliferation in osteosarcoma," Tumour Biology, vol. 37, no. 7, pp. 9441-9450, 2016.

[6] L. Qiu, Q. Tang, G. Li, and K. Chen, "Long non-coding RNAs as biomarkers and therapeutic targets: Recent insights into hepatocellular carcinoma," Life Sciences, vol. 191, pp. 273282, 2017.

[7] H. Lei, Y. Gao, and X. Xu, "LncRNA TUG1 influences papillary thyroid cancer cell proliferation, migration and EMT formation through targeting miR-145," Acta Biochimica et Biophysica Sinica, vol. 49, no. 7, pp. 588-597, 2017.

[8] A. Tahiri, K. Røe, A. H. Ree et al., "Differential inhibition of exvivo tumor kinase activity by vemurafenib in BRAF(V600E) and BRAF wild-type metastatic malignant melanoma," PLoS One, vol. 8, no. 8, article e72692, 2013.

[9] S. X. Yuan, J. Wang, F. Yang et al., "Long noncoding RNA DANCR increases stemness features of hepatocellular 
carcinoma by derepression of CTNNB1," Hepatology, vol. 63, no. 2, pp. 499-511, 2016.

[10] C. A. Yang, S. Bauer, Y. C. Ho, F. Sotzny, J. G. Chang, and C. Scheibenbogen, "The expression signature of very long non-coding RNA in myalgic encephalomyelitis/chronic fatigue syndrome," Journal of Translational Medicine, vol. 16, no. 1, p. 231, 2018.

[11] Z. Tang, C. Li, B. Kang, G. Gao, C. Li, and Z. Zhang, "GEPIA: a web server for cancer and normal gene expression profiling and interactive analyses," Nucleic Acids Research, vol. 45, no. W1, pp. W98-W102, 2017.

[12] I. Diboun, L. Wernisch, C. A. Orengo, and M. Koltzenburg, "Microarray analysis after RNA amplification can detect pronounced differences in gene expression using limma," BMC Genomics, vol. 7, no. 1, p. 252, 2006.

[13] Y. Zhai, Y. Li, J. Zhang et al., "Identification of the gene cluster for bistropolone-humulene meroterpenoid biosynthesis in Phoma sp.," Fungal Genetics and Biology, vol. 129, pp. 7-15, 2019.

[14] R. D. Page, TreeView, Glasgow University, Glasgow, UK, 2001.

[15] X. Hu, G. Shen, X. Lu, G. Ding, and L. Shen, "Identification of key proteins and lncRNAs in hypertrophic cardiomyopathy by integrated network analysis," Archives of Medical Science, vol. 15, no. 2, pp. 484-497, 2019.

[16] X. Shi, T. Huang, J. Wang et al., "Next-generation sequencing identifies novel genes with rare variants in total anomalous pulmonary venous connection," eBioMedicine, vol. 38, pp. 217-227, 2018.

[17] D. Zhang, P. Zhang, P. Yang et al., "Downregulation of ATP1A1 promotes cancer development in renal cell carcinoma," Clinical Proteomics, vol. 14, no. 1, p. 15, 2017.

[18] Z. Jin, J. W. Chung, W. Mei et al., "Regulation of nuclearcytoplasmic shuttling and function of Family with sequence similarity 13, member A (Fam13a), by B56-containing PP2As and Akt," Molecular Biology of the Cell, vol. 26, no. 6, pp. 11601173, 2015.

[19] X. B. Wang, H. Wang, H. Q. Long, D. Y. Li, and X. Zheng, "LINC00641 regulates autophagy and intervertebral disc degeneration by acting as a competitive endogenous RNA of miR-153-3p under nutrition deprivation stress," Journal of Cellular Physiology, vol. 234, no. 5, pp. 7115-7127, 2019.

[20] Z. Cui, X. An, J. Li, Q. Liu, and W. Liu, "LncRNA MIR22HG negatively regulates miR-141-3p to enhance DAPK1 expression and inhibits endometrial carcinoma cells proliferation," Biomedicine \& Pharmacotherapy, vol. 104, pp. 223-228, 2018.

[21] S. Talukdar, S. K. Das, A. K. Pradhan et al., "Novel function of MDA-9/Syntenin (SDCBP) as a regulator of survival and stemness in glioma stem cells," Oncotarget, vol. 7, no. 34, pp. 54102-54119, 2016.

[22] C. Song, J. Zhang, Y. Liu et al., "Construction and analysis of cardiac hypertrophy-associated lncRNA-mRNA network based on competitive endogenous RNA reveal functional lncRNAs in cardiac hypertrophy," Oncotarget, vol. 7, no. 10, pp. 10827-10840, 2016.

[23] R. Hu, W. Liu, H. Li et al., “A Runx2/miR-3960/miR-2861 regulatory feedback loop during mouse osteoblast differentiation," Journal of Biological Chemistry, vol. 286, no. 14, pp. 12328-12339, 2011.

[24] W. Zhao, H. Fu, S. Zhang, S. Sun, and Y. Liu, "LncRNA SNHG16 drives proliferation, migration, and invasion of hem- angioma endothelial cell through modulation of miR-520d3p/STAT3 axis," Cancer Medicine, vol. 7, no. 7, pp. 33113320, 2018.

[25] W. Lu, Y. Xu, J. Xu, Z. Wang, and G. Ye, "Identification of differential expressed lncRNAs in human thyroid cancer by a genome-wide analyses," Cancer Medicine, vol. 7, no. 8, pp. 3935-3944, 2018.

[26] H. Huang, J. Chen, C. M. Ding, X. Jin, Z. M. Jia, and J. Peng, "LncRNA NR2F1-AS1 regulates hepatocellular carcinoma oxaliplatin resistance by targeting ABCC1 via miR-363," Journal of Cellular and Molecular Medicine, vol. 22, no. 6, pp. 3238-3245, 2018.

[27] K. W. Chung, S. W. Kim, and S. W. Kim, "Gene expression profiling of papillary thyroid carcinomas in Korean patients by oligonucleotide microarrays," Journal of the Korean Surgical Society, vol. 82, no. 5, pp. 271-280, 2012.

[28] Y. Xu, Y. Zheng, H. Liu, and T. Li, "Modulation of IGF2BP1 by long non-coding RNA HCG11 suppresses apoptosis of hepatocellular carcinoma cells via MAPK signaling transduction," International Journal of Oncology, vol. 51, no. 3, pp. 791-800, 2017. 